\title{
Highly Focused Supersonic Microjets
}

\author{
Yoshiyuki Tagawa, ${ }^{1, *}$ Nikolai Oudalov, ${ }^{1}$ Claas Willem Visser, ${ }^{1}$ Ivo R. Peters, ${ }^{1}$ Devaraj van der Meer, ${ }^{1}$ Chao Sun, ${ }^{1, \dagger}$ \\ Andrea Prosperetti, ${ }^{1,2}$ and Detlef Lohse ${ }^{1, \neq}$ \\ ${ }^{1}$ Physics of Fluids Group, MESA+ Institute and Faculty of Science and Technology, University of Twente, \\ P.O. Box 217, 7500 AE Enschede, Netherlands \\ ${ }^{2}$ Department of Mechanical Engineering, Johns Hopkins University, Baltimore, Maryland 21218, USA
}

(Received 11 December 2011; published 9 July 2012)

\begin{abstract}
This paper describes the production of thin, focused microjets with velocities of up to $850 \mathrm{~m} / \mathrm{s}$ by the rapid vaporization of a small mass of liquid in an open liquid-filled capillary. The vaporization is caused by the absorption of a low-energy laser pulse. A likely explanation of the observed phenomenon is based on the impingement of the shock wave caused by the nearly instantaneous vaporization on the free surface of the liquid. We conduct an experimental study of the dependence of the jet velocity on several parameters and develop a semiempirical relation for its prediction. The coherence of the jets and their high velocity, good reproducibility, and controllability are unique features of the system. A possible application is to development of needle-free drug-injection systems that would be of great importance for health care worldwide.
\end{abstract}

DOI: 10.1103/PhysRevX.2.031002

Subject Areas: Fluid Dynamics

\section{INTRODUCTION}

Transient liquid jets produced by flow focusing have been studied from various perspectives due to fundamental interest and their widespread occurrence (see, e.g., the review article by Eggers and Villermaux [1]). Common instances include bubbles bursting at a liquid surface $[2,3]$, high-amplitude Faraday waves [4-8], jets generated by the impulsive acceleration of a liquid surface [9], shaped charges, and others.

In all these cases, the jet is produced when a liquid surface, concave toward the gas, is impulsively accelerated. The resulting "kinematic focusing" of the liquid, which converges toward the center of the curvature, is responsible for the peculiarly high-speed jets generated in this way.

In shaped charges, this convergence is caused by a detonation wave investing the free surface. The early history of these devices and the principle on which they operate is described by Birkhoff et al. [10], followed by many works, e.g., Curtis and Kelly [11], and Petit et al. [12] in more recent years. The focusing can, however, also be caused by geometrical effects simply due to the concave shape of the interface without reliance on liquid compressibility (see, e.g., [13-18]).

In this study, we describe yet another method of generating such a jet. We use a microtube in which the impulsive

\footnotetext{
*y.tagawa@utwente.nl

c.sun@utwente.nl

‡.lohse@utwente.nl
}

Published by the American Physical Society under the terms of the Creative Commons Attribution 3.0 License. Further distribution of this work must maintain attribution to the author(s) and the published article's title, journal citation, and DOI. acceleration of the interface is due to the abrupt vaporization of a small mass of liquid caused by the absorption of a laser pulse.

Bubble generation by the absorption of a focused laser pulse in the bulk of a liquid volume has been an established experimental technique for a long time (see, e.g., $[19,20]$ ). More recently, the same technique has been used to generate bubbles in the proximity of the free surface of a liquid [21-24]. In this case, strong jets are often observed. For example, Thoroddsen et al. [24], who focused a laser pulse into a drop resting on a glass surface, report jet maximum velocities of $250 \mathrm{~m} / \mathrm{s}$ produced by small bubbles near the free surface but without the ability to control the number, size, and location of these bubbles.

In the present work, we also use a laser pulse to create a bubble near a free surface, but the geometry of the system and conditions of the experiment are quite different from those of earlier workers. Furthermore, the microjet that we describe has extraordinary characteristics: It is created under controlled conditions with good reproducibility; its maximum velocity reaches $850 \mathrm{~m} / \mathrm{s}$, which in air is a Mach number approaching 3; its diameter is typically 1 order of magnitude smaller than the diameter of the capillary tube; and its generation requires moderate laser energies of the order of $10 \mu \mathrm{J}$, comparable to the typical energy of a laser pointer.

A particularly attractive potential application of the present supersonic microjets is for needle-free drug injection [25]. For this application, in which a liquid solution containing a drug is forced to penetrate human or animal tissue through the skin, ultrahigh velocities (greater than $200 \mathrm{~m} / \mathrm{s}$ ), fine scales (down to $30 \mu \mathrm{m}$ ), and good reproducibility and controllability are essential.

The development of needle-free drug-injection systems is of paramount importance in the global fight against 
the spread of disease, as these systems help to prevent contamination by needles. Several methods of microjet generation suitable for this application have been proposed [26,27], but they all have several shortcomings: The production of each jet requires a significant amount of energy, the resulting shape of the ejected liquid mass tends to be diffuse rather than focused, the small nozzles are easily clogged, and others.

The microjets produced by the method described in this study are free from these problems. In particular, they are faster and highly focused. Furthermore, the avoidance of very thin nozzles makes the systems less susceptible to clogging and thus improves the reliability and controllability.

The experimental setup is presented in Sec. II, followed by a description of jet formation and evolution in Sec. III. The quantitative dependencies of the jet speed on the various control parameters are discussed in Sec. IV, culminating in the derivation of a scaling law for the jet velocity as a function of the various parameters. The paper ends with a summary and conclusions in Sec. V.

\section{EXPERIMENTAL SETUP AND CONTROL PARAMETERS}

Figure 1(a) shows a sketch of the whole experimental setup, which is similar to that used by Sun et al. [28]. One end of the capillary tube is connected to a syringe pump (Model PHD 2000, Harvard Apparatus, USA) containing a
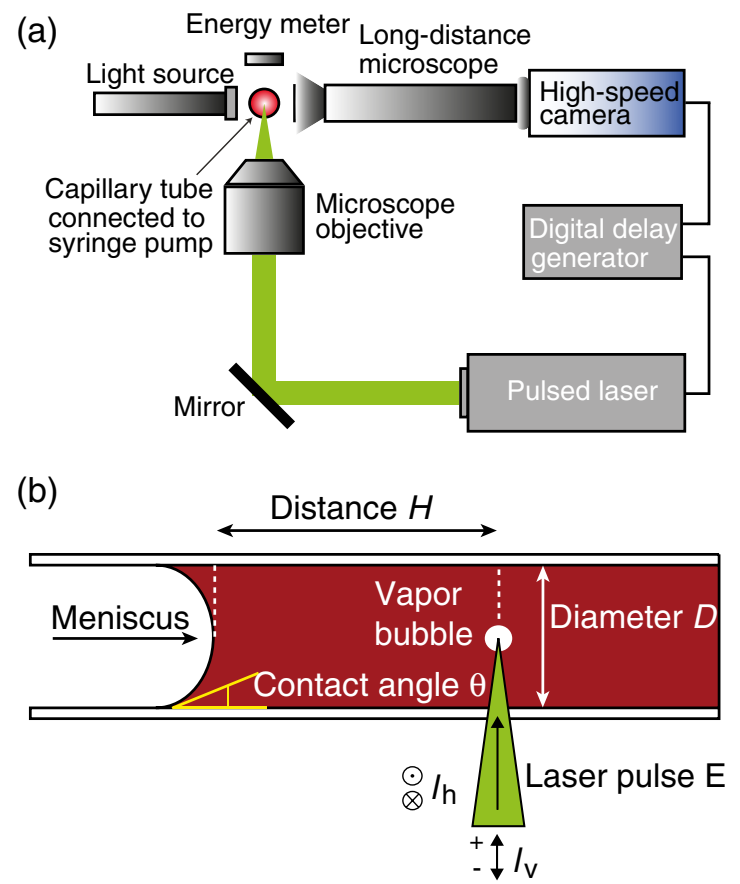

FIG. 1. (a) A sketch of the experimental setup; the capillary tube is aligned perpendicular to the graph. (b) Definition of experimental parameters with a side view of the capillary in which the microjet formation takes place. water-based red dye. The other end is open to the air. A 532-nm, 6-ns laser pulse (100-mJ Nd:YAG laser, Solo PIV, New Wave Research, USA) is focused through a $10 \times$ microscope objective to a point inside the capillary. The laser energy is monitored by means of an energy meter (Gentec-EO XLE4 or Gentec-EO QE12SP-S-MT-D0, Canada) placed behind the capillary. The energy absorbed by the liquid is calibrated by measuring the difference between the readings of the meter when the glass tube is filled with dyed and clear water. The jet formation is recorded using high-speed cameras with a frame rate of up to $10^{6} \mathrm{fps}$ (HPV-1, Shimadzu Corporation, Japan, and FASTCAM SA1.1, Photron, USA). The minimum interframe and exposure times are $1 \mu \mathrm{s}$ and $250 \mathrm{~ns}$, respectively. This system enables us to observe the capillary from below using a microscope (Axiovert 40 CFL, Carl Zeiss, Germany). A long-distance microscope (Model K2, Infinity, USA) with a maximum magnification of $12 \times$ is connected to the camera in order to capture the jet formation from the side and vary the field of view by adjusting the magnification. Illumination for the camera is provided by a fiber lamp (ILP-1 Light Source, Olympus, Japan), emitting light that passes through the filter protecting the camera. A digital delay generator (Model 555, Berkeley Nucleonics Corporation, USA) is used to synchronize the camera and the laser. Images are analyzed with tracking software.

The experimental parameters are indicated in Fig. 1(b) together with a side view of the capillary. The distance between the meniscus and the laser focus position is $H ; E$ is the absorbed laser energy; $\theta$ is the initial contact angle of the liquid with the glass capillary, which has a diameter $D$; $l_{\mathrm{v}}$ is the distance offset of the laser focus with respect to the capillary axis along a vertical diameter, positive when the focus is beyond the tube axis; $l_{\mathrm{h}}$ is the focus offset of the laser perpendicular to the tube axis and the diameter along which $l_{\mathrm{v}}$ is measured. Borosilicate-glass capillary tubes (Capillary Tube Supplies Limited, UK) of three different sizes are used in the experiments, with inner diameters $D$ of 50,200 , and $500 \mu \mathrm{m}$ and outer diameters of 80,220 , and $520 \mu \mathrm{m}$, respectively. The tip of the capillary tube is dipped in a hydrophobic solution $(1 \mathrm{H}, 1 \mathrm{H}, 2 \mathrm{H}, 2 \mathrm{H}-\mathrm{Perfluorooctyltrichlorosilane})$ to pin the

TABLE I. The range of control parameters for the initial contact angle of the liquid with the glass capillary $\theta$, the distance between the meniscus and the laser focus position $H$; the absorbed laser energy $E$; the focus offset of the laser in the vertical plane $l_{\mathrm{v}}$; and the focus offset of the laser in the horizontal plane $l_{\mathrm{h}}$. The inner diameters of capillary tubes $D$ are 50, 200 , and $500 \mu \mathrm{m}$.

\begin{tabular}{lrrrrrl}
\hline \hline & $\theta$ & $E(\mu \mathrm{J})$ & $H(\mu \mathrm{m})$ & $D(\mu \mathrm{m})$ & $l_{\mathrm{v}}(\mathrm{mm})$ & $l_{\mathrm{h}}(\mathrm{mm})$ \\
\hline Lower limit & $20^{\circ}$ & 19 & 200 & 50 & -1 & 0 \\
Upper limit & $90^{\circ}$ & 880 & 2500 & 500 & 2.5 & 0.1 \\
\hline \hline
\end{tabular}



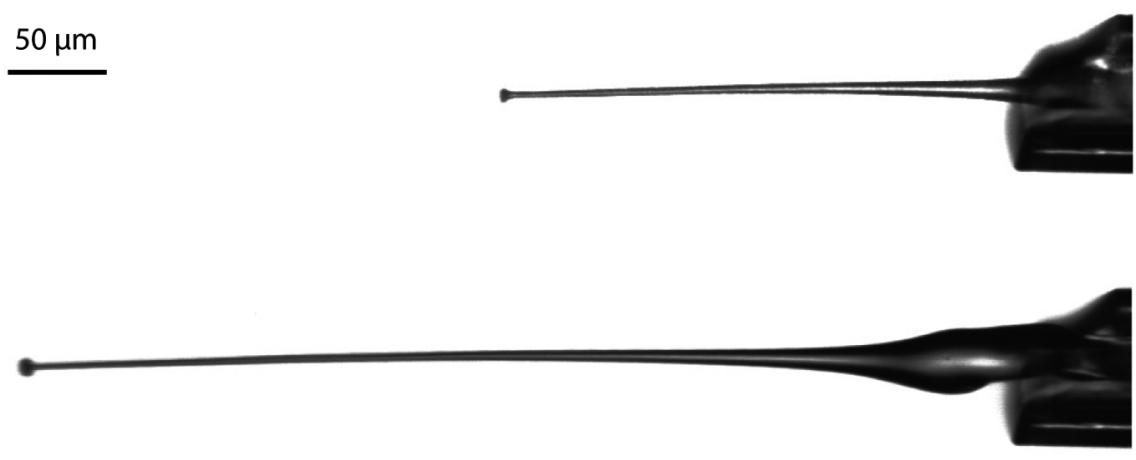

FIG. 2. Images of a supersonic microjet generated in a 50- $\mu \mathrm{m}$ capillary tube. The capillary is visible on the right side; the jet tip is shown on the left. The jet travels from right to left with a speed of $490 \mathrm{~m} / \mathrm{s}$. Time between images is $500 \mathrm{~ns}$.

contact line and permit control of the contact angle $\theta$ between $20^{\circ}$ and $90^{\circ}$. For uncoated tubes, the initial contact angle is found to be $25^{\circ} \pm 3^{\circ}$, which is similar to the data reported by Sumner et al. [29] for borosilicate glass. The range of the control parameters is summarized in Table I.

\section{JET FORMATION AND EVOLUTION}

An example of a supersonic jet visualized using the technique of dual-flash illumination by laser-induced fluorescence [30] is shown in Fig. 2. The capillary is on the right, the jet tip is on the left, and the jet travels from right to left with a speed of $490 \mathrm{~m} / \mathrm{s}$. The tip of this jet has grown into an almost spherical mass which will eventually become detached as a droplet. At the other end, the jet is thicker and slower.

Figures 3(a) and 3(b) show successive frames [31] taken with the Shimadzu camera of the jet produced in a 500- $\mu \mathrm{m}$ tube by using laser energies of $365 \mu \mathrm{J}$ and $650 \mu \mathrm{J}$. The bubble growth is larger in the second case, and the motion of the liquid column in the tube is more evident. The use of this larger capillary produces relatively slow jets with asymptotic velocities of about $21 \mathrm{~m} / \mathrm{s}$ and $55 \mathrm{~m} / \mathrm{s}$, respectively, which facilitates the visualization. The open end of the tube is on the left; the other end is connected to the syringe pump as explained before. The bubble is generated near the lower wall of the capillary and expands mainly in the direction of the open end. In spite of this off-center position and asymmetric growth, the jet maintains an axisymmetric shape with a sharp tip. Its diameter is always much smaller than that of the capillary. In this instance, it is about $50 \mu \mathrm{m}$, about 10 times smaller than the capillary. The beginning of the instability that eventually leads to droplet pinch-off can be discerned. We have always found that, unless the bubble is generated less than one diameter away from the free surface $(H \leq D)$, the asymmetry of its growth does not affect the axial symmetry of the jet. This observation supports the hypothesis that the cause of the thin jet is the sudden onset of a high pressure rather than the bubble expansion per se.
Figure 4(a) shows another typical sequence of jet evolution [32]. The corresponding history of the jet-tip velocity is shown in Fig. 4(b), and it is seen to be nonmonotonic. Immediately after the laser pulse, the interface sets into motion, shown in image (i). The maximum velocity $V_{\max }$ occurs when a well-formed jet just begins to appear, as shown in image (ii), by which time the meniscus has lost the initial concave shape. This feature is observed irrespective of the jet speed and is therefore compatible with the hypothesis of kinematic focusing due to the initial concave shape of the interface. The subsequent deceleration shown in image (iii) is due to surface tension, as suggested by the fact that the deceleration is suppressed

(a)

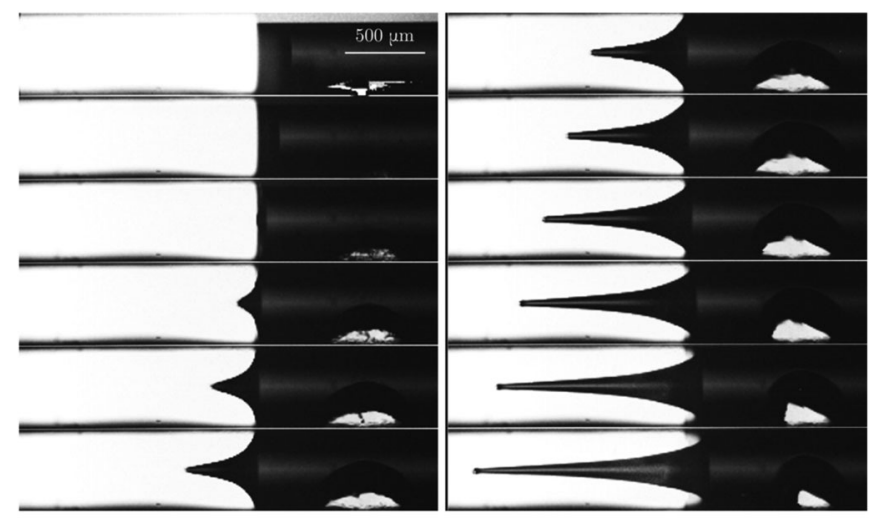

(b)
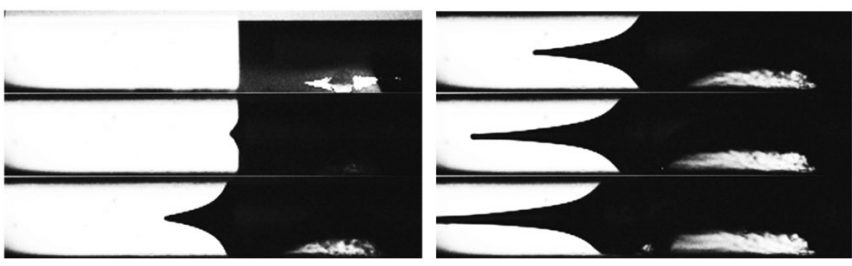

FIG. 3. Bubble growth and jet evolution after focusing the laser on the axis of a $500-\mu \mathrm{m}$ capillary tube with (a) $E=365 \mu \mathrm{J}$ and $H=600 \mu \mathrm{m}$ and (b) $E=650 \mu \mathrm{J}$ and $H=600 \mu \mathrm{m}$. The first image shows the tube at the moment the laser is fired. The subsequent images are taken $7 \mu$ s apart. (See Ref. [31] for the related movie.) 
(a)

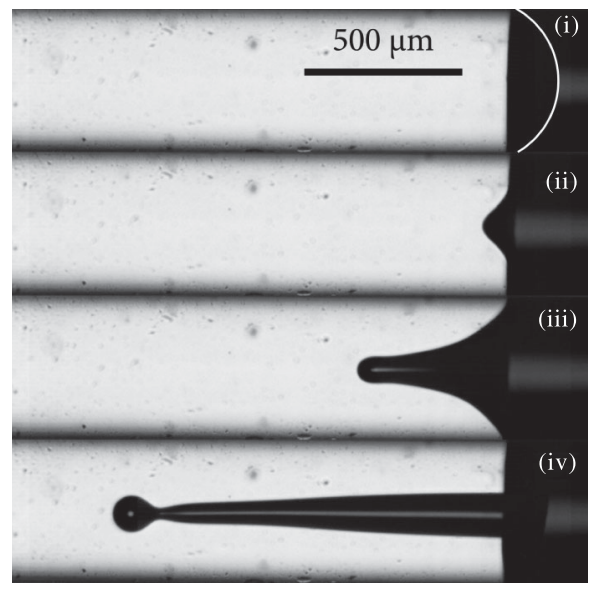

(b)

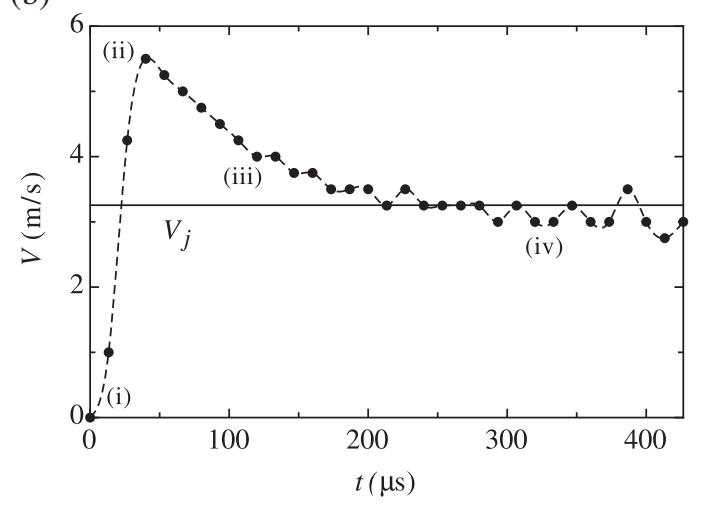

FIG. 4. (a) Jet evolution after focusing the laser in a 500- $\mu \mathrm{m}$ capillary tube. The first image shows the tube at the moment that the laser is fired. The subsequent images are taken at later stages, each corresponding to a point in time shown in (b). (See Ref. [32] for the related movie.) (b) Velocity of the jet tip in a $500-\mu \mathrm{m}$ tube as a function of time after the laser is fired.

at higher velocities, i.e., at higher Weber numbers. As the jet becomes thinner, the rate of new surface generation decreases, surface tension becomes less important, and the jet tip velocity reaches an asymptotic value $V_{j}$; shortly thereafter, a drop starts to form at the tip, shown in image (iv), and it eventually becomes detached.

It is worth mentioning that a very small fraction of the liquid receiving the initial impulse and focusing into the jet is in contact with the wall, and therefore its motion is not significantly affected by contact-line phenomena. In any event, since the process is very rapid, such phenomena can only affect a thin liquid layer of the order of the viscous penetration length, which can be estimated as less than $5 \mu \mathrm{m}$. Most of the liquid is therefore in an essentially inertia-dominated, nearly inviscid motion and is not significantly affected by contact-line phenomena.

\section{PARAMETER DEPENDENCE}

In this section, we study the dependence of the jet speed on various control parameters.

\section{A. Contact angle $\theta$}

Since the concavity of the meniscus is crucial for the jet formation, the first parameter we investigate is the initial radius of curvature $R_{0}$ of the free surface. This quantity, which was determined by fitting a circle to the image of the free surface, is related to the contact angle $\theta$ of the liquid with the capillary by the formula

$$
R_{0}=\frac{D}{2 \cos \theta}
$$

which is valid because the effect of gravity in this system is negligible, as shown by the smallness of the Bond number $\mathrm{Bo}=\rho g R_{0}^{2} / \gamma$ (where $\rho$ is the liquid density, $\gamma$ the surface tension coefficient, and $g$ the acceleration of gravity), typically of the order of $10^{-3}$.

The initial radius of curvature is varied by minor adjustments of the liquid volume by means of the syringe. For these tests, the laser focus is on the tube axis, and the energy $E$ and the distance $H$ between focus position and meniscus are kept constant at $460 \pm 20 \mu \mathrm{J}$ and $460 \pm 40 \mu \mathrm{m}$, respectively; experiments are carried out on $500-\mu \mathrm{m}$ diameter tubes.

Figure 5 shows four sequences [33] taken with different initial contact angles, with three snapshots per sequence. The shape of the jet is significantly influenced by the radius of curvature. These results show that the more curved the meniscus shape (smaller radius of curvature and lower initial contact angle), the higher the jet velocity due to the increased focusing. For increasing initial contact angles, the jet becomes thicker and less focused. Eventually, when the initial contact angle is $90^{\circ}$ or larger, the focusing is lost, no jet is formed, and the liquid is only pushed out as a plug by the expanding bubble.

These results confirm that the initial shape of the meniscus plays an important role for the acceleration of the jet, as discussed in Sec. III. For a quantitative analysis, we derive a

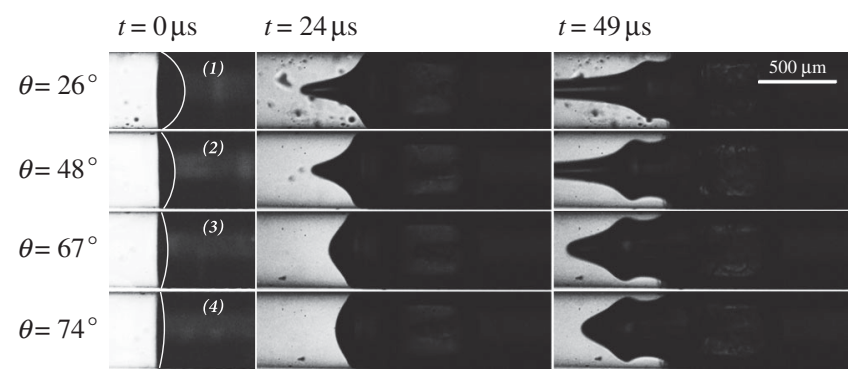

FIG. 5. Images showing the effect of varying the initial contact angle $\theta$ on the jet shape: (1) $\theta=26^{\circ}$, (2) $\theta=48^{\circ}$, (3) $\theta=67^{\circ}$, and (4) $\theta=74^{\circ}$. The first image (left column) shows the liquid in the capillary prior to the laser pulse, illustrating the initial contact angle and meniscus shape. The second image in each set (center column) is taken $24 \mu \mathrm{s}$ after the laser pulse, and the third image (right column) follows $25 \mu$ s later. ((See Ref. [33] for the four related movies.) 
scaling of the velocity increment due to the flow focusing as follows.

We assume that, due to the initial high-pressure pulse, the liquid acquires a velocity $V_{0}$ normal to the initial free surface, and the surface tension is negligible. After a short time $\Delta t$, the free surface is still spherical with a smaller radius $R_{0}-V_{0} \Delta t$. The new velocity $V_{0}+\Delta V$ acquired due to this geometrical focusing can be estimated from the principle of mass conservation as

$$
\left(V_{0}+\Delta V\right)\left(R_{0}-V_{0} \Delta t\right)^{2}=V_{0} R_{0}^{2} .
$$

Neglecting a higher-order term, we obtain the scale of the velocity increment as

$$
\Delta V \sim \frac{V_{0}^{2} \Delta t}{R_{0}} .
$$

Since the focusing effect is caused by the liquid particles approaching the axis of the tube, it occurs on a characteristic length scale of the order of the mean distance between the free surface of the liquid and the axis, which is of the order of the tube diameter $D$. Thus, the time scale $\Delta t$ for the geometrical focusing can be estimated as

$$
\Delta t \sim \frac{D}{V_{0}} .
$$

This estimate is supported by the fact that, as described in Sec. III, the acceleration due to the focusing occurs approximately until the meniscus loses the concave shape. Thus, upon combining Eqs. (1), (3), and (4), the increase in velocity due to flow focusing can be estimated as

$$
\Delta V \sim V_{0} \cos \theta .
$$

Thus, one may expect the velocity $V_{j}$ resulting from the focusing to be given by

$$
V_{j} \sim V_{0}+\Delta V=V_{0}(1+\beta \cos \theta) .
$$

As shown in Fig. 6, this relation provides a reasonable fit to the data taken in the 500- $\mu \mathrm{m}$ tube by taking $\beta \simeq 13$ and $V_{0} \simeq 2.5 \mathrm{~m} / \mathrm{s}$. A more elaborated model will be described in Peters et al. [34]. The curve $1-\sin \theta$ suggested by Antkowiak et al. [9] is also shown in Fig. 6. Although, unlike the previous model which estimates the asymptotic jet velocity, the latter model is aimed to describe the initial velocity of the jet, its trend agrees with the present experimental data.

If the initial velocity $V_{0}$ is due to the reflection of a shock wave from the free surface, the strength $\Delta p$ of the shock can be estimated as $\Delta p=1 / 2 \rho c V_{0}$, where $c$ is the speed of sound in the liquid. With the previous value of $V_{0}$, we find $\Delta p \sim 20$ atm.

The initial velocity for the incompressible model can be estimated by assuming that it is acquired during a short time $\Delta \tau$ by the action of an overpressure $\Delta p$. If the laser focus is at a distance $H$ from the free surface, this argument leads us to $H \rho V_{0} \sim \Delta p \Delta \tau$ [35]. With

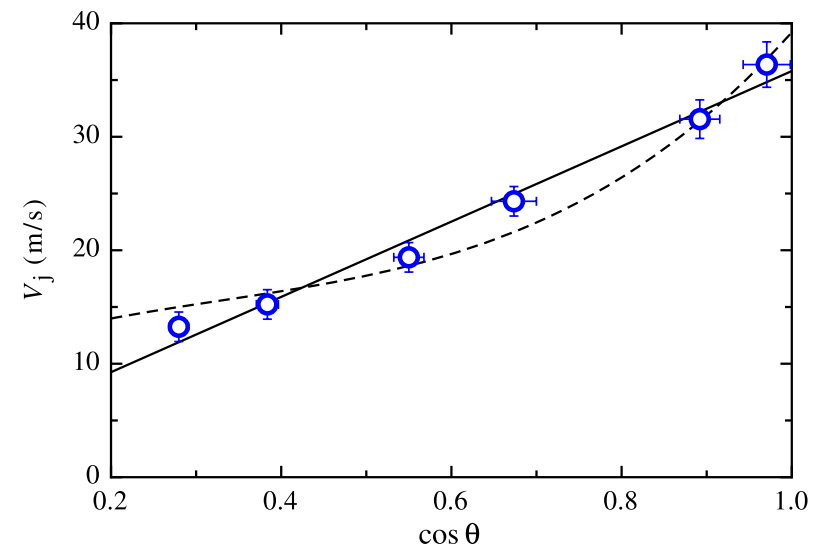

FIG. 6. Asymptotic jet velocity $V_{\mathrm{j}}$ as a function of the cosine of the initial contact angle. The solid line is a linear fit to the data. The dashed line is a $1-\sin \theta$ fit to the data suggested by Antkowiak et al. [9].

$H=460 \mu \mathrm{m}, V_{0}=2.5 \mathrm{~m} / \mathrm{s}$, and $\Delta \tau=6 \mathrm{~ns}$, this estimate gives $\Delta p \sim 2000 \mathrm{~atm}$, which is larger than the critical pressure of water which equals approximately 218 atm. For higher-velocity cases, the incompressible pressure estimate increases considerably [34].

\section{B. Distance $\boldsymbol{H}$ between laser focus and free surface}

Figure 7 shows the experimental results for the asymptotic jet tip velocity $V_{\mathrm{j}}$, defined in Fig. 4, as a function of the distance $H$ between the laser spot and the free surface [see Fig. 1(b)]. Two different capillary tubes with diameters of $200 \mu \mathrm{m}$ and $500 \mu \mathrm{m}$ were used, and two different energy levels $E$ for each: $165 \mu \mathrm{J}$ and $232 \mu \mathrm{J}$, and $305 \mu \mathrm{J}$

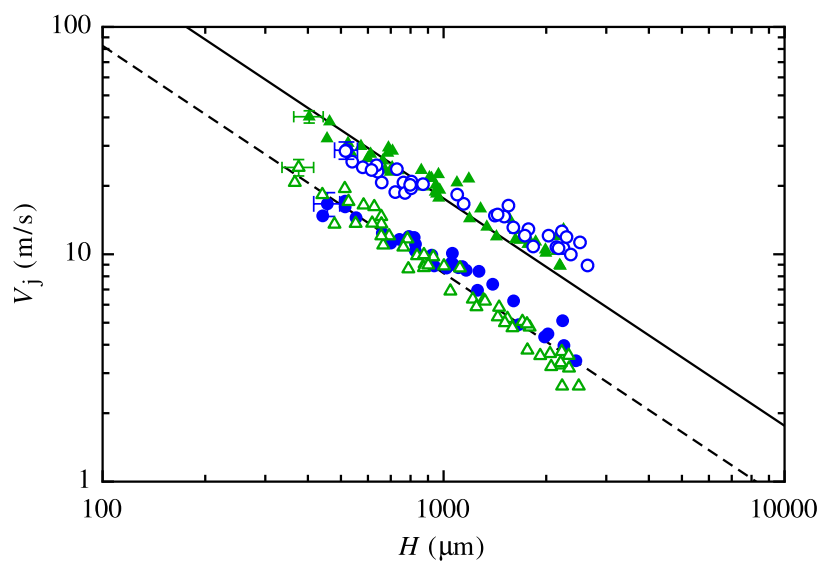

FIG. 7. Asymptotic jet velocity $V_{\mathrm{j}}$ as a function of the distance $H$ between the laser spot and the free surface for different capillary diameters and energies. The triangles represent the data for the 200- $\mu \mathrm{m}$ tube at $E=232 \mu \mathrm{J}(\boldsymbol{\Delta})$ and $E=165 \mu \mathrm{J}$ $(\triangle)$; the circles show the results for the $500-\mu \mathrm{m}$ tube at $E=458 \mu \mathrm{J}(\bigcirc)$ and $E=305 \mu \mathrm{J}(\bigcirc)$. The solid and dashed lines show a -1 power law. Typical error bars are shown for a few data points. 
and $458 \mu \mathrm{J}$, respectively. The data show that, for both tube diameters, $V_{\mathrm{j}}$ is inversely proportional to the distance $H$ by about a factor of 10 . This dependence is particularly well satisfied at the lower energy levels.

It seems possible to rationalize this observation on the basis of both the incompressible-flow-focusing model and the shock-wave model. In the framework of the former, one may note that the mass of the liquid slug set into motion is approximately proportional to $H$, and therefore so is its inertia. In the framework of the shockwave mechanism, on the other hand, we recall that the pressure amplitude of a locally generated shock wave in a free fluid decreases inversely with the distance. In our conditions, the fluid is confined within the tube, but the size of the initially vaporized liquid is at least 1 order of magnitude smaller than the tube diameter so that this geometrical attenuation may play a role. Another possibility is that there is viscous attenuation of the shock due to the no-slip condition at the tube wall (see, e.g., $[36,37])$. Whatever the reason for the decreasing shock pressure with increasing distance $H$, given that the experiments show that the velocity is inversely proportional to the distance, this suggests that the velocity is proportional to the pressure.

\section{Absorbed energy $E$}

The next parameter of interest is the energy absorbed by the liquid. For this part of the study, the laser focus is on the tube axis, and its distance from the meniscus was kept constant at $410 \pm 40 \mu \mathrm{m}, 390 \pm 40 \mu \mathrm{m}$, and $600 \pm$ $40 \mu \mathrm{m}$ for the $50-\mu \mathrm{m}, 200-\mu \mathrm{m}$, and $500-\mu \mathrm{m}$ diameter tubes, respectively.

Figure 8(a) shows the asymptotic jet velocity $V_{\mathrm{j}}$ vs the absorbed energy for different tube diameters. The data are well fitted by a linear function with a positive intercept at $V_{\mathrm{j}}=0$. This feature embodies the existence of a threshold $E_{\text {heat }}$, below which no jet is formed. The threshold values for the $50-\mu \mathrm{m}, 200-\mu \mathrm{m}$, and $500-\mu \mathrm{m}$ capillary tubes are approximately $20 \mu \mathrm{J}, 100 \mu \mathrm{J}$, and $200 \mu \mathrm{J}$, respectively. Thus, the threshold value is an increasing function of the tube diameter $D$. It is interesting to note that, for the 50- $\mu \mathrm{m}$ capillary tube, the linear relation between $V_{j}$ and $E-E_{\text {heat }}$ is preserved even when the jet speed becomes supersonic, as shown in Fig. 8(b). The absorbed energy for all cases is of the order of $109 \mathrm{~J} / \mathrm{m}^{3}$, which is of the same order as the vaporization enthalpy of water at normal conditions.

As noted before, in the framework of the shock wave model, the experimental results of Sec. IV B suggest that the velocity is proportional to the pressure. This linear relation then implies that the pressure of the shock wave at the meniscus is proportional to the absorbed energy. From the incompressible-flow viewpoint, one may conclude that the initial impulse imparted to the liquid is proportional to the absorbed energy.
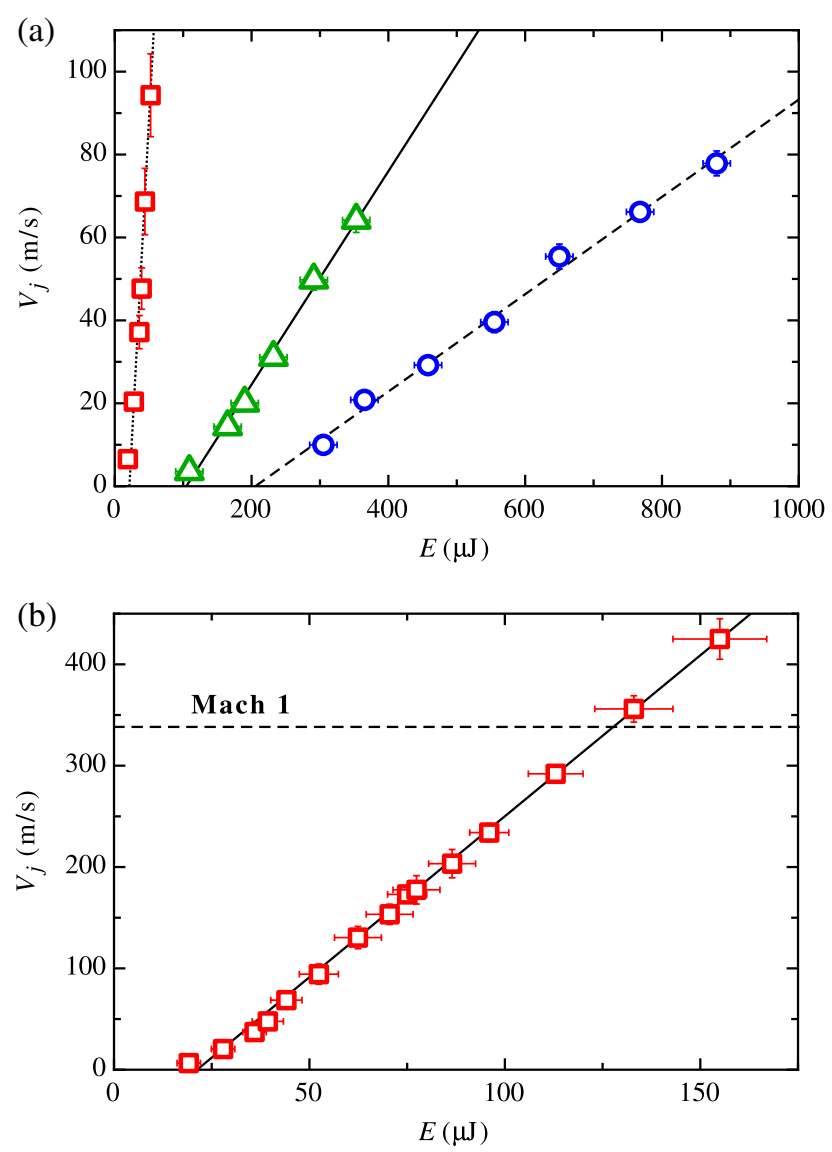

FIG. 8. (a) Asymptotic jet velocity $V_{\mathrm{j}}$ as a function of the energy absorbed by the liquid in the capillary tubes. The squares $(\square)$, triangles $(\triangle)$ and circles $(\bigcirc)$ represent the data for capillary tubes with $50-\mu \mathrm{m}, 200-\mu \mathrm{m}$, and $500-\mu \mathrm{m}$ inner diameters, respectively. Each data point is the result of at least three measurements. The lines are linear fits to the data. (b) The data for the $50-\mu \mathrm{m}$ diameter tube are shown on an expanded scale. Each data point is the result of at least five measurements. The linear dependence on the energy is seen to hold also for supersonic speeds.

The slopes of the data for the 50- $\mu \mathrm{m}, 200-\mu \mathrm{m}$, and $500-\mu \mathrm{m}$ diameter tubes are $3.09,0.26$, and $0.12 \mathrm{~m} /(\mathrm{s} \cdot \mu \mathrm{J})$, respectively. Thus, for the same absorbed energy, the jet speed decreases with increasing tube diameter. The effects of the diameter $D$ on the jet velocity are discussed further in Sec. IV D.

In Fig. 8(b), no data points at higher $V_{j}$ could be acquired because, in the current configuration, the glass tube breaks at the point where the laser focuses when the energy $E$ is too high.

\section{Diameter of microcapillary $D$}

In the study of the dependence of the jet velocity on the tube diameter, it was necessary to adjust the energy ranges depending on the diameter. On the basis of the results reported above, we have concluded that, to a good accuracy, 
$V_{j}$ is proportional to $\left(E-E_{\text {heat }}\right)$ and $(1+\beta \cos \theta)$ and inversely proportional to $H$. We express this dependency by writing

$$
V_{\mathrm{j}} \simeq f(D) \frac{\left(E-E_{\text {heat }}\right)(1+\beta \cos \theta)}{H},
$$

where the prefactor $f(D)$ embodies the dependence on the diameter. By trying a power-law dependence, $f(D)=$ $f_{0} D^{-\alpha}$, the data is best fit by $\alpha \simeq-1.14$, which may be considered close to -1 .

Especially the data for the $200-\mu \mathrm{m}$, and $500-\mu \mathrm{m}$ tubes agree well with the line of slope -1 , while that for $50-\mu \mathrm{m}$ is above the line with slope -1 . This could be attributed to the fact that different effects, such as laser focusing, start to play an important role at smaller tube sizes due to the higher curvature of the tube. According to the impulse pressure description suggested by Antkowiak et al. [9] and the analysis leading to Eq. (5), the diameter of the tube should not play a role. A possible reason for this apparent dependence is that the same energy delivered to a smaller-diameter tube will give rise to a faster jet due to a smaller inertia. To strengthen this hypothesis, it may be noted that, if the kinetic energy is constant for a given absorbed energy $E$, since the mass of the accelerated liquid is approximately proportional to $D^{2}$, it follows that the velocity would decrease proportionally to $D^{-1}$. From the results that were presented in the previous sections, we derive the following empirical law:

$$
V_{\mathrm{j}} \simeq C_{0} \frac{\left(E-E_{\text {heat }}\right)(1+\beta \cos \theta)}{H D} .
$$

Calculating $C_{0}$ by fitting the data in Fig. 9, we get $C_{0}=$ $0.0027(\mathrm{~Pa} \cdot \mathrm{s})^{-1}$. It is interesting to note that $C_{0}$ has the

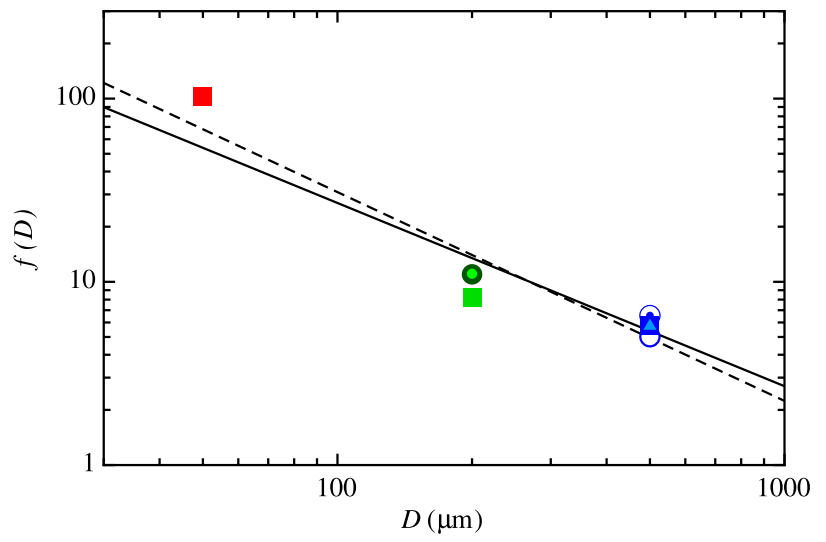

FIG. 9. Prefactor $f(D)$ from Eq. (7) vs the capillary diameter. The circles, squares, and diamonds are constants obtained from the energy-dependence, distance-dependence, and initial-contact angle-dependence experiments, respectively. The value of the prefactor increases with smaller capillaries. A best-fit power law (dashed line) with power -1.14 is shown as well as a solid line with power -1 as shown in Eq. (8), which shows less agreement with the data, particularly for small $D$. dimensions of an inverse dynamic viscosity. This feature appears to be accidental, as viscous effects are not expected to play a significant role, as noted before.

\section{E. Focus offsets $l_{\mathrm{v}}$ and $l_{\mathrm{h}}$}

The sensitivity of the jet to the laser focus position relative to the capillary axis was studied by displacing it in the horizontal $\left(l_{\mathrm{h}}\right)$ and vertical $\left(l_{\mathrm{v}}\right)$ directions [see Fig. 1(b)]. The results are shown in Fig. 10.

Figure 10(a) indicates that the jet velocity decreases monotonically for increasing $l_{\mathrm{h}}$ all other conditions being held constant. We hypothesize that this trend is explained by a reduced volume of the vaporized liquid. To test this hypothesis, the jet velocity is compared to the initial laser-induced vaporized volume as estimated with the geometrical optics approximation described in Appendix A. As shown in Fig. 10(a), the jet velocity roughly scales with the calculated volume of the liquid vaporized by the laser pulse.
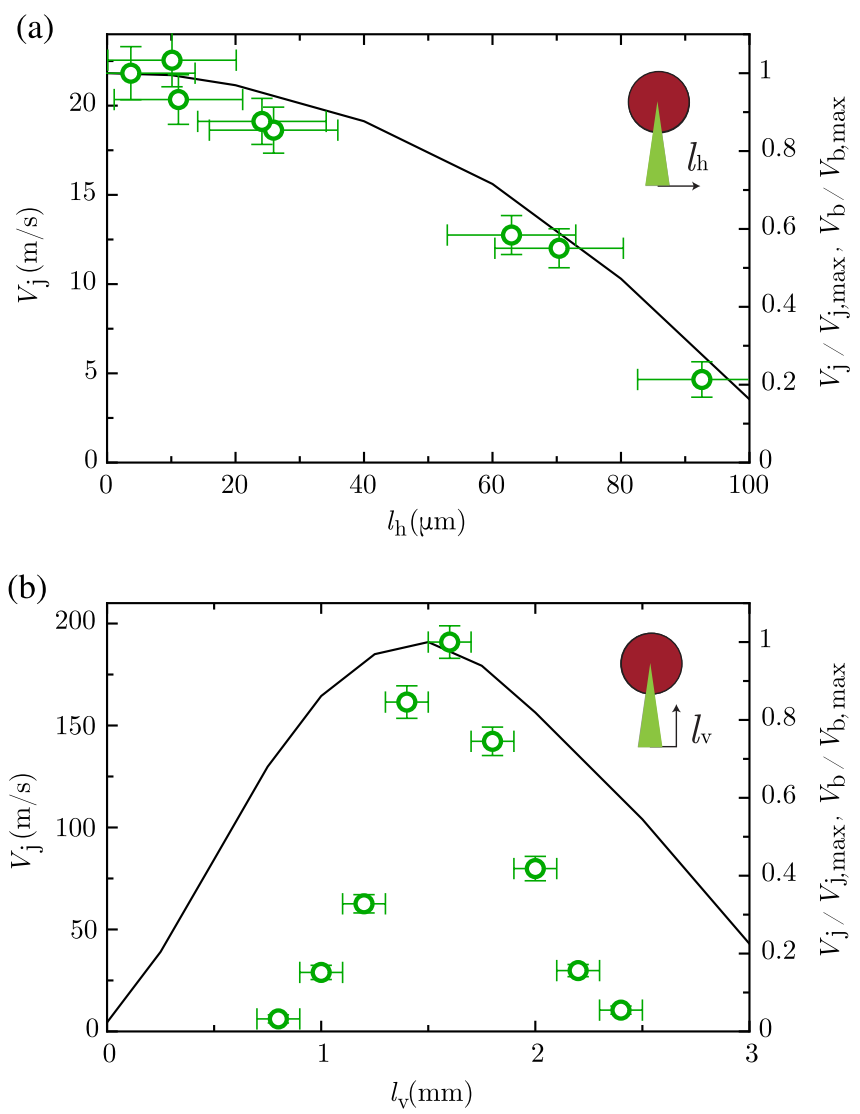

FIG. 10. (a) Asymptotic jet velocity $V_{\mathrm{j}}(\bigcirc)$ as a function of the horizontal focus displacement of the laser for the $200-\mu \mathrm{m}$ tube. The black curve shows the normalized vaporized-liquid volume determined by geometrical-optics approximation in both graphs (right y axis). (b) Asymptotic jet velocity $V_{\mathrm{j}}$ as a function of the vertical focus displacement of the laser. The inserts show the capillary and the laser focus (triangle) and the directions of $l_{\mathrm{h}}$ and $l_{\mathrm{v}}$ as seen from the capillary opening. 
Figure 10(b) shows the jet speed vs the distance of the focus from the axis of the tube $l_{\mathrm{v}}$. The jet velocity increases with increasing $l_{\mathrm{v}}$ up to $l_{\mathrm{v}} \approx 1.5 \mathrm{~mm}$ and then decreases for larger $l_{\mathrm{v}}$. The rise is likely caused by the larger area of the capillary-tube surface illuminated by the laser, which increases as $l_{\mathrm{v}}$ is increased by moving the laser toward the tube. As the vaporization always occurs at the capillary wall, a larger vapor mass will be created by increasing $l_{\mathrm{v}}$. However, when the laser is moved too close to the capillary, the energy-per-unit area of the laser beam decreases and eventually drops below the threshold required for bubble formation. This situation was also modeled by geometrical optics. As shown in Fig. 10(b), the maximum jet speed and maximum bubble size are found at the same offset. Even though the overall trend is the same, the normalized values of the vaporized volume deviate from those of the jet velocity. This difference is not surprising since there is no reason to think that vaporized volume and jet growth are linearly related to each other. The analysis of these aspects is beyond the scope of this work; they will be studied in future investigations.

The velocity increase with increasing $l_{\mathrm{v}}$ shown in Fig. 10(b) is also found for other tube diameters, as shown in Fig. 11, where the jet speed vs the vertical focus offset $l_{\mathrm{v}}$ is plotted for different diameters down to $50 \mu \mathrm{m}$. Jets with a velocity up to $850 \mathrm{~m} / \mathrm{s}$ could be consistently produced with the smallest capillaries. Measurements beyond this velocity could not be obtained, as the gradual increase of the absorbing liquid volume with increasing $l_{\mathrm{v}}$ eventually leads to such a violent bubble expansion that the capillary breaks. We also observed that, at the higher energies, the wall-shear stresses exerted by the liquid pushed out of the capillary by the expanding bubble after the thin jet formation is so large as to shear off the capillary tip. It is

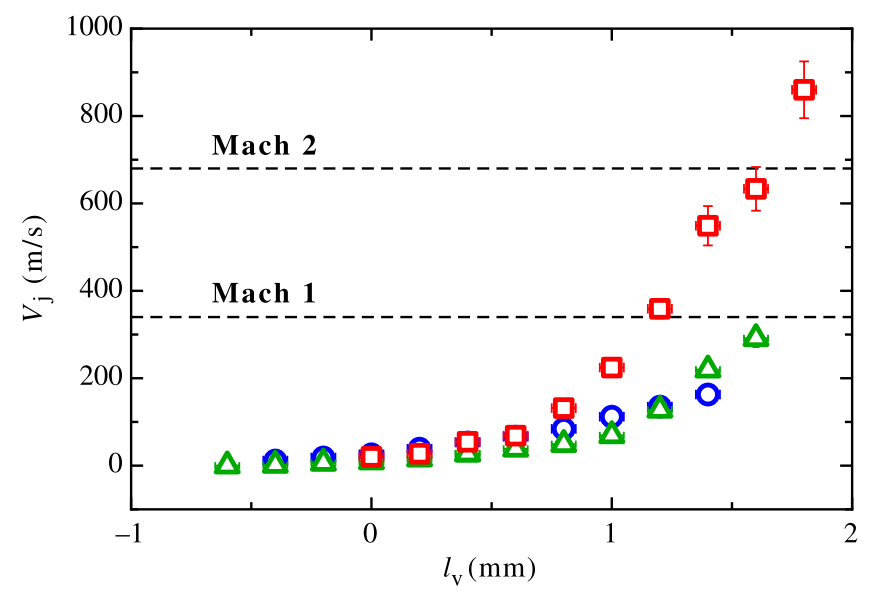

FIG. 11. Asymptotic jet velocity $V_{\mathrm{j}}$ as a function of the focus offset of the laser for different capillary diameters. The circles (O) represent measurements for the 500- $\mu \mathrm{m}$ tube; the triangles $(\triangle)$ refer to the $200-\mu \mathrm{m}$ tube; and the squares $(\square)$ are for the $50-\mu \mathrm{m}$ tube. Each data point is the result of at least three measurements. possible that, with different materials, the velocity of the jet can be increased. When the jet velocity approaches the speed of sound in water, some limitations to further increases may arise due to the dominance of compressibility effects.

\section{SUMMARY AND CONCLUSIONS}

The dynamics of the high-speed microjet generated by a laser-induced rapid vaporization of water in a microtube has been studied. It has been shown that the jets so generated can reach speeds as high as $850 \mathrm{~m} / \mathrm{s}$ with good controllability.

The dependence of the jet velocity upon various controlling parameters has been investigated in a series of experiments the results of which have been summarized in the empirical relationship provided in Eq. (8). This equation shows the effects of the distance between the laser focus and the liquid meniscus at the mouth of the microtube, the absorbed laser energy, the liquid-tube initial contact angle, and the tube diameter.

The jet velocity exhibits an inverse proportionality to the tube diameter and to the distance of the laser focus from the free surface, while it is proportional to the absorbed energy above a threshold value. The velocity is very strongly dependent on the curvature of the free surface, which is a function of the liquid-tube initial contact angle. It is also critically dependent on the amount of liquid vaporized and on its distance from the free surface, both of which can be varied by varying the position of the laser focus. In particular, it has been found that the offset of the laser focus with respect to the tube axis has a strong and very nontrivial effect. To elucidate the origin of this result, we have used a geometrical-optics construction coupled with the Beer-Lambert law to determine approximately the size of the region where vaporization occurs and the energy is absorbed. While still preliminary, the results of this analysis are in general agreement with the data. Further theoretical and numerical investigations of the jetting phenomenon will be addressed in Peters et al. [34].

A priori results of the paper can be interpreted according to two different scenarios: one in which the shock wave generated by the nearly instantaneous vaporization plays a dominant role, and one in which the phenomenon is essentially incompressible. The estimate of the overpressure provided for the relatively low-velocity case in Sec. IV C suggests that, according to the incompressible model, a much higher pressure is necessary to account for the observations as compared to the shock-wave model. For higher-velocity cases, the incompressible overpressure becomes even much larger than the critical pressure of water [34]. These considerations lend support to the shock-wave interpretation of the results presented in this paper.

The insights gained through this research and the ability to generate focused, controllable, and high-velocity 
microjets open new doors for the realization, among others, of reliable needlefree drug-delivery systems.

\section{ACKNOWLEDGMENTS}

We thank Rory Dijkink for starting the experiments. We also thank Christophe Clanet, Alexander Klein, and Gerben Morsink for helpful discussions. We gratefully acknowledge the support of this work by FOM (Stichting voor Fundamenteel Onderzoek der Materie).

\section{APPENDIX A: ESTIMATE OF THE LIQUID ENERGY ABSORPTION BY GEOMETRICAL OPTICS}

A geometrical-optics approximation was developed to analyze energy absorption of the laser-beam energy absorption by the liquid. The three main components of this calculation are: (1) splitting up the Gaussian laser beam into many different rays, each one with a representative energy; (2) following the path of each ray through the capillary, including (full or partial) wall reflections; (3) using the Beer-Lambert law to model the local energy absorbed by the liquid. This law describes the local irradiance $I$, and is given by $I=I_{0} \times 10^{-\epsilon s}$, with $I_{0}$ the beam irradiance at the cylindrical outer surface of the tube; $\epsilon$ the absorption coefficient; and $s$ the arc length along the partial of the ray propagating in the liquid. The absorption coefficient was measured as $\epsilon=84 \times 10^{3} \mathrm{~m}^{-1}$; the local energy loss of a ray equals $\partial I / \partial s$.

The liquid volumes are discretized into cells constituting a grid around the central loss area. The local energy lost by each ray in each cell was calculated using the BeerLambert law and added to the liquid in the cell. Subsequently, for each cell, the total energy absorbed over the duration of the light pulse was compared with an estimate of the energy necessary for evaporation at room conditions, namely, $E_{\text {boil }}=\rho\left(C_{p} \Delta T+\Delta H_{\text {vap }}\right) \Delta V_{\mathrm{c}}$, with $\rho$ the liquid density; $\Delta T=80 \mathrm{~K}$ the difference between the initial liquid temperature and the boiling temperature; $C_{\mathrm{p}}=4181 \mathrm{~J} /(\mathrm{kgK})$ the heat capacity at constant pressure; $\Delta H_{\text {vap }}=2.26 \mathrm{MJ} / \mathrm{kg}$ the vaporization enthalpy; and $\Delta V_{\mathrm{c}}$ the cell volume. If the energy absorbed by a cell exceeded $E_{\text {boil }}$, the cell volume was assumed to be vaporized instantaneously. Summation of the vaporized-cell volumes then provided a measure for the initial bubble volume and position.

[1] J. Eggers and E. Villermaux, Physics of Liquid Jets, Rep. Prog. Phys. 71, 036601 (2008).

[2] L. Duchemin, S. Popinet, C. Josserand, and S. Zaleski, Jet Formation in Bubbles Bursting at a Free Surface, Phys. Fluids 14, 3000 (2002).
[3] M.S. Longuet-Higgins, Bubbles, Breaking Waves and Hyperbolic Jets at a Free Surface, J. Fluid Mech. 127, 103 (1983), published online in 2006.

[4] C.L. Goodridge, H.G.E. Hentschel, and D.P. Lathrop, Breaking Faraday Waves: Critical Slowing of Droplet Ejection Rates, Phys. Rev. Lett. 82, 3062 (1999).

[5] B. W. Zeff, B. Kleber, J. Fineberg, and D.P. Lathrop, Singularity Dynamics in Curvature Collapse and Jet Eruption on a Fluid Surface, Nature (London) 403, 401 (2000).

[6] M. S. Longuet-Higgins, Vertical Jets from Standing Waves, Proc. R. Soc. A 457, 495 (2001).

[7] M. S. Longuet-Higgins and D. G. Dommermuth, Vertical Jets from Standing Waves. II, Proc. R. Soc. A 457, 2137 (2001).

[8] M. S. Longuet-Higgins and D. G. Dommermuth, On the Breaking of Standing Waves by Falling Jets, Phys. Fluids 13, 1652 (2001).

[9] A. Antkowiak, N. Bremond, S. Le Dizès, and E. Villermaux, Short-Term Dynamics of a Density Interface Following an Impact, J. Fluid Mech. 577, 241 (2007).

[10] G. Birkhoff, D. P. MacDougall, E. M. Pugh, and G. Taylor, Explosives with Lined Cavities, J. Appl. Phys. 19, 563 (1948).

[11] J.P. Curtis and R. J. Kelly, Circular Streamline Model of Shaped-Charge Jet and Slug Formation with Asymmetry, J. Appl. Phys. 75, 7700 (1994).

[12] J. Petit, V. Jeanclaude, and C. Fressengeas, Breakup of Copper Shaped-Charge Jets: Experiment, Numerical Simulations, and Analytical Modeling, J. Appl. Phys. 98, 123521 (2005).

[13] S. Gekle, J. M. Gordillo, D. van der Meer, and D. Lohse, High-Speed Jet Formation after Solid Object Impact, Phys. Rev. Lett. 102, 034502 (2009).

[14] S. Gekle and J. M. Gordillo, Generation and Breakup of Worthington Jets after Cavity Collapse. Part 1. Jet Formation, J. Fluid Mech. 663, 293 (2010).

[15] R. Bergmann, D. van der Meer, M. Stijnman, M. Sandtke, A. Prosperetti, and D. Lohse, Giant Bubble Pinch-Off, Phys. Rev. Lett. 96, 154505 (2006)

[16] M. S. Longuet-Higgins and H. Oguz, Critical Microjets in Collapsing Cavities, J. Fluid Mech. 290, 183 (1995), published online in 2006.

[17] R. Bergmann, D. van der Meer, S. Gekle, A. van der Bos, and D. Lohse, Controlled Impact of a Disk on a Water Surface: Cavity Dynamics, J. Fluid Mech. 633, 381 (2009).

[18] S. T. Thoroddsen, T. G. Etoh, and K. Takehara, Experiments on Bubble Pinch-Off, Phys. Fluids 19, 042101 (2007).

[19] W. Lauterborn, T. Kurz, R. Mettin, and C.D. Ohl, Experimental and Theoretical Bubble Dynamics, Adv. Chem. Phys. 110, 295 (1999).

[20] A. Vogel and V. Venugopalan, Mechanisms of Pulsed Laser Ablation of Biological Tissues, Chem. Rev. 103, 577 (2003).

[21] I. Apitz and A. Vogel, Material Ejection in Nanosecond ErYAG Laser Ablation of Water, Liver, and Skin, Appl. Phys. A 81, 329 (2005). 
[22] D. Obreschkow, P. Kobel, N. Dorsaz, A. de Bosset, C. Nicollier, and M. Farhat, Cavitation Bubble Dynamics inside Liquid Drops in Microgravity, Phys. Rev. Lett. 97, 094502 (2006).

[23] E. Robert, J. Lettry, M. Farhat, P. A. Monkewits, and F. Avellan, Cavitation Bubble Behavior inside a Liquid Jet, Phys. Fluids 19, 067106 (2007).

[24] S. T. Thoroddsen, K. Takehara, T. G. Etoh, and C. D. Ohl, Spray and Microjets Produced by Focusing a Laser Pulse into a Hemispherical Drop, Phys. Fluids 21, 112101 (2009).

[25] S. Mitragotri, Current Status and Future Prospects of Needle-Free Liquid Jet Injectors, Nat. Rev. Drug Discov. 5, 543 (2006).

[26] T. Han and J.J. Yoh, A Laser Based Reusable Microjet Injector for Transdermal Drug Delivery, J. Appl. Phys. 107, 103110 (2010).

[27] V. Menezes, S. Kumar, and K. Takayama, Shock Wave Driven Liquid Microjets for Drug Delivery, J. Appl. Phys. 106, 086102 (2009).

[28] C. Sun, E. Can, R. Dijkink, D. Lohse, and A. Prosperetti, Growth and Collapse of a Vapour Bubble in a Microtube: The Role of Thermal Effects, J. Fluid Mech. 632, 5 (2009).

[29] A. L. Sumner, E. J. Menke, Y. Dubowski, J. T. Newberg, R. M. Penner, J.C. Hemminger, L.M. Wingen, T. Brauers, and B.J. Finlayson-Pitts, The Nature of Water on Surfaces of Laboratory Systems and Implications for
Heterogeneous Chemistry in the Troposphere, Phys. Chem. Chem. Phys. 6, 604 (2004).

[30] A. van der Bos, A. Zijlstra, E. Gelderblom, and M. Versluis, iLIF: illumination by Laser-Induced Fluorescence for Single Flash Imaging on a Nanoseconds Timescale, Exp. Fluids 51, 1283 (2011).

[31] To view the movie that was the source for Fig. 3, see Supplemental Material at http://link.aps.org/supplemental/ 10.1103/PhysRevX.2.031002.

[32] To view the movie that was the source for Fig. 4(a), see Supplemental Material at http://link.aps.org/supplemental/ 10.1103/PhysRevX.2.031002.

[33] To view the four movies that were the sources for Figs. 5(a)-5(d), see Supplemental Material at http://link .aps.org/supplemental/10.1103/PhysRevX.2.031002.

[34] I. R. Peters, Y. Tagawa, N. Oudalov, C. Sun, D. van der Meer, A. Prosperetti, and D. Lohse (unpublished).

[35] E. Ory, H. Yuan, A. Prosperetti, S. Popinet, and S. Zaleski, Growth and Collapse of a Vapor Bubble in a Narrow Tube, Phys. Fluids 12, 1268 (2000).

[36] G. Mirshekari and M. Brouillette, One-Dimensional Model for Microscale Shock Tube Flow, Shock Waves 19, 25 (2009).

[37] D. Ngomo, A. Chaudhuri, A. Chinnayya, and A. Hadjadj, Numerical Study of Shock Propagation and Attenuation in Narrow Tubes Including Friction and Heat Losses, Comput. Fluids 39, 1711 (2010). 